\title{
Anderson Localization in Carbon Nanotubes: Defect Density and Temperature Effects
}

\author{
Blanca Biel, ${ }^{1}$ F. J. García-Vidal, ${ }^{1, *}$ Angel Rubio, ${ }^{2,3,4,5}$ and Fernando Flores ${ }^{1}$ \\ ${ }^{1}$ Departamento de Física Teórica de la Materia Condensada, Universidad Autónoma de Madrid, E-28049 Madrid, Spain \\ ${ }^{2}$ Institut für Theoretische Physik, Freie Universität Berlin, Arnimallee 14, D-14195 Berlin, Germany \\ ${ }^{3}$ Departamento de Física de Materiales, Facultad de Ciencias Químicas, UPV/EHU, Centro Mixto CSIC-UPV/EHU, San Sebastián, \\ Spain \\ ${ }^{4}$ Donostia International Physics Center, E-20018 San Sebastián, Spain \\ ${ }^{5}$ European Theoretical Spectroscopy Facility \\ (Received 29 July 2005; published 19 December 2005)
}

\begin{abstract}
The role of irradiation induced defects and temperature in the conducting properties of single-walled $(10,10)$ carbon nanotubes has been analyzed by means of a first-principles approach. We find that divacancies modify strongly the energy dependence of the differential conductance, reducing also the number of contributing channels from two (ideal) to one. A small number of divacancies (5-9) brings up strong Anderson localization effects and a seemly universal curve for the resistance as a function of the number of defects. It is also shown that low temperatures, about $15-65 \mathrm{~K}$, are enough to smooth out the fluctuations of the conductance without destroying the exponential dependence of the resistivity as a function of the tube length.
\end{abstract}

DOI: 10.1103/PhysRevLett.95.266801

The ubiquity of defects in materials science limits somehow the performance of the material; this is even more dramatic when the dimensionality of the system is reduced moving towards nanostructures, in particular, carbon nanotubes [1]. Understanding and controlling the conductance of these systems is decisive for their possible application in molecular devices [2-9]. In perfect single-walled carbon nanotubes (SWNTs), electrons propagate ballistically if the inelastic processes can be neglected, i.e., if the electronic phase coherence length, $L_{\phi}$, is larger than the nanotube length, $L$. High quality metallic SWNTs exhibit $L_{\phi}$ as large as $1 \mu \mathrm{m}$ [4]. If inelastic processes, like the electron-phonon interaction, are important, the system conducts within the diffusive regime. On the other hand, it has been shown $[7,8]$ that defects induce Anderson localization in the electron states of a SWNT if $L_{0}<L<$ $L_{\phi}, L_{0}$ being the localization length. This is the regime we are interested in.

This Anderson localization phenomenon is due to the concerted action of quantum confinement and defect scattering effects $[10,11]$. The theoretical calculations presented in Ref. [8] were concentrated on the zerotemperature case. Temperature $(T)$ adds new dephasing mechanisms for the coherent electron transport in SWNTs that should be considered on the same footing as the other sources of dephasing. In this context, a fully microscopic study of the electronic transport in defected SWNT in the Anderson localization regime taking into account the effect of finite $T$ is lacking. This is the goal of this Letter: to fully understand the properties of the localization regime as a function of the nanowire length, temperature, density of defects, and strength of the defect scattering potential for the paradigmatic $(10,10)$ chirality.

As we are interested in modeling the role of defects in the conductivity of SWNTs, we have chosen to analyze in
PACS numbers: 73.63.Fg, 72.10.Fk, 73.23.-b

detail the monovacancies and divacancies that are the most common defects that appear upon atom irradiation [9]; the comparison of these two cases will allow us to analyze the electron localization and its effect on the nanotube conductance as a function of the different scattering potentials associated with those defects. As our calculations of the zero-bias conductance drop associated with a single defect show a conductance of $1.98 G_{0}$ for the monovacancy and $1.36 G_{0}$ for the lateral divacancy $\left(2 G_{0}=4 e^{2} / h\right.$ is the conductance of an ideal nanotube), divacancies will show a more dramatic effect in the nanotube conductance [8]. Accordingly, although we present results for monovacancies and divacancies in order to draw more general conclusions, most of our discussion concentrates on the divacancy case.

The conductance calculations are done within a firstprinciples local-orbital density functional (LODF) method that maps the full Hamiltonian into a local-orbital one, allowing the use of the standard machinery of nonequilibrium Green's function in order to extract the transmission probability, and from that the nanotube conductance [12]. The simulation geometry consists of a device region with the defected nanotubes connected to two semiinfinite perfect tubes. The geometry of the nanotube around each vacancy is calculated using this LODF method, and the defected nanotube is defined by adding $N$ defects separated by a mean distance $d$. Then, the length of the nanotube, $L$, is $L=N \times d$. The defects are assumed to be distributed along the rim of the tube with a distance between consecutive defects (measured along the tube axis) presenting a uniform random distribution between 0 and $2 d$. The conductance calculations are performed for a statistically significant number of defect realizations. For the sake of simplicity, we present results for a nanotube with either only lateral divacancies (this is the most favor- 
able geometry for divacancies [8]) or with only monovacancies. In our calculations, we obtain an effective localorbital Hamiltonian associated with the $s p^{3}$-basis set of the fireball orbitals [13]; details about the technique can be found in Ref. [8]. Because of the local nature of this approach, it is feasible to calculate the electronic properties of very long nanotubes (up to several microns long) with an arbitrary distribution of defects.

First we analyze the energy dependence of the nanotube differential conductance, $g(E)$, for two different densities of defects and zero $T$. In Fig. 1 we plot $g(E)$ of a $(10,10)$ carbon nanotube for four random configurations of defects with two different interdefect distances $(d=45.4$ and $75.5 \mathrm{~nm}$ ) and two numbers of defects (15 and 25) introduced in the tube, typical values observed in the experiments [8]. In all cases we observe strong fluctuations of the differential conductance as a function of energy: this is a clear indication of the localized nature of the electronic states. Notice also, comparing Figs. 1(a) and 1(b), that $g(E)$ also depends strongly on $d$. For finite $T$ calculations the crucial point to realize is that electrons are injected into the nanotube with energies within a window of the order of

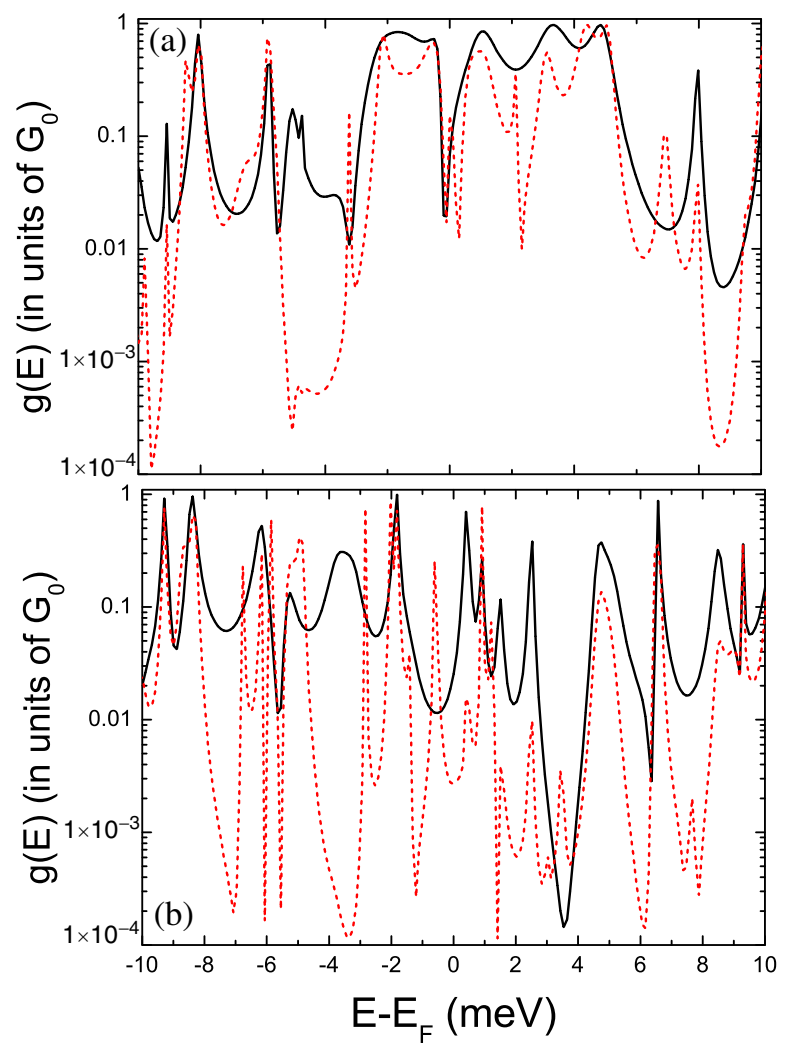

FIG. 1 (color online). Differential conductance for two defected single-wall carbon nanotubes. (a) The distance between defects of $d=45.4 \mathrm{~nm}$ and (b) for $d=75.5 \mathrm{~nm}$. Two different number of defects (15, continuous lines, and 25, dashed lines) are analyzed in both panels. Notice that the plotted energy window is smaller than the room temperature energy window (25 meV).
$k_{B} T$. Then, it is expected that the strong fluctuations in the conductance would be washed out for a finite $T$. This will happen if the thermal coherent length $L_{T}\left(L_{T}=\hbar v_{\mathrm{F}} / k_{B} T\right.$, $v_{\mathrm{F}}$ being the Fermi velocity) is smaller than $L_{0}$. One naively says also that Anderson localization could not appear in this limit. This would be the case only if $L_{\phi}$ is of the order of $L_{0}$. As perfect metallic tubes exhibit a ballistic response over long distances (microns), the electrons injected in the nanotube with different energies do not suffer inelastic scattering events, and they behave as independent electrons [14]. In this picture, the total conductance is just the sum of many contributions from electrons injected within the thermal energy window [15].

In Fig. 2(a) we show the dependence of the nanotube resistance with the tube length for different temperatures and a given random distribution of divacancies. These curves have been calculated as follows. First, we evaluate the resistance of the longest tube (corresponding to $N=25$ defects in this case) with a given random configuration of divacancies. Next, we calculate the resistance of a nanotube with $N-1$ defects just eliminating the last divacancy [the length of the nanotube is then reduced
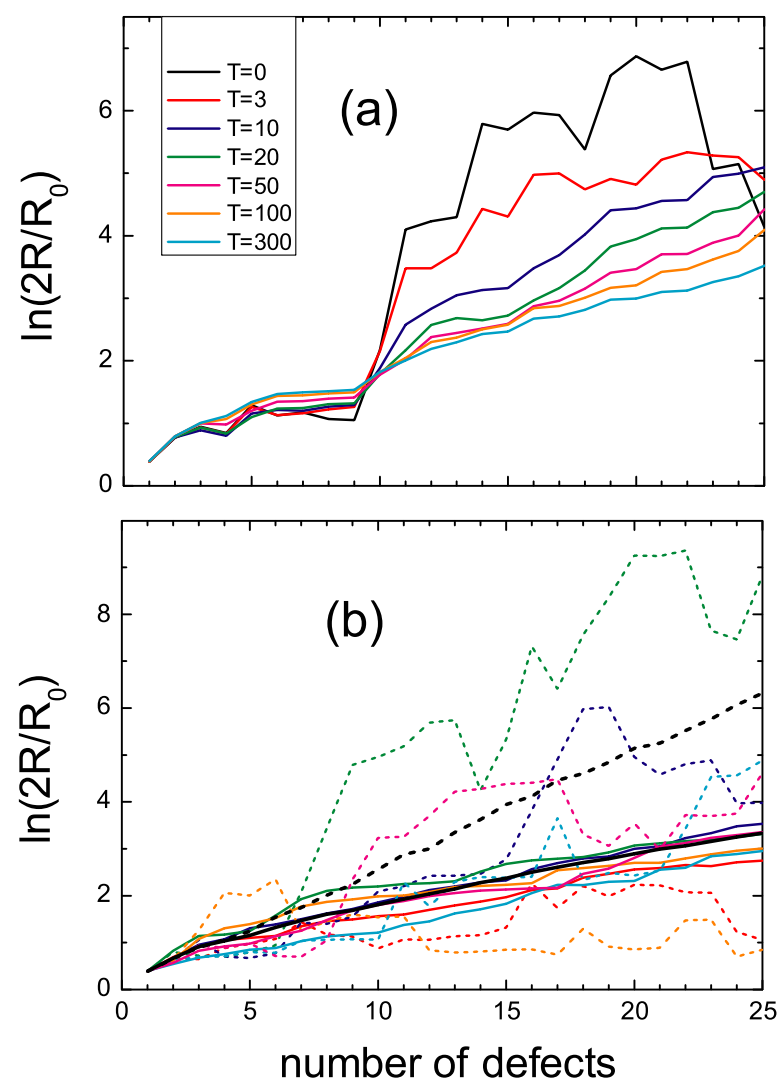

FIG. 2 (color online). (a) Temperature dependence of the calculated resistance for a defected nanotube with $d=$ $75.5 \mathrm{~nm}$. (b) For $T=0$ (dashed lines) and $T=300 \mathrm{~K}$ (continuous curves) we plot the calculated resistances for different random defect configurations with $d=75.5 \mathrm{~nm}$. The mean value of the resistances is also plotted in black. 
to $L=(N-1) d]$. This procedure is repeated until we end with a nanotube containing only one divacancy. Then, the whole calculation is repeated for several temperatures but with the same random configuration of defects. At zero $T$, as expected, the fluctuations in the resistance are very strong. The effect of temperature is striking: at room $T$, there is a complete reduction of the random fluctuations seen at $T=0 \mathrm{~K}$. However, we still get the exponential increase of resistance with tube length that points out that the Anderson localization regime survives even at room $T$. Figure 2(a) also demonstrates that only a very low $T$ (less than $20 \mathrm{~K}$ ) is needed to wash out the fluctuations of the resistance as a function of the tube length. This result explains why fluctuations are not seen at room $T$ as experimentally reported in [8]. In Fig. 2(b) we plot both the zero and room $T$ resistances for several random configurations of divacancies with $d=75.5 \mathrm{~nm}$. Notice that at room $T$ the resistance of different configurations is very insensitive to the particular random distribution of divacancies. This is not the case for $T=0 \mathrm{~K}$ where there are strong fluctuations with respect to the average value.

Figure 3 shows our calculations for the mean value of the room $T$ resistance (as a result of an average over 15 random cases, as explained above) as a function of the carbon nanotube length for different $d$ 's. Our results indicate that this room $T$ resistance seems to fit a universal curve once it is plotted in terms of the number of defects $(N)$ instead of the total length $(L)$. An important result of performing a finite $T$ calculation is that the resistance curves of Fig. 3 fit better as $R_{0} \times \exp \left(L / L_{0}\right)$ than as $R_{0} / 2 \times \exp \left(L / L_{0}\right)\left(R_{0}\right.$ being $\left.1 / G_{0}\right)$, for $N>3-5$. This is a strong indication of the reduction of the conducting channels induced by both defects and finite $T[16,17]$; only one channel is contributing to the total conductance. Moreover, this also suggests that the nanotube enters in the

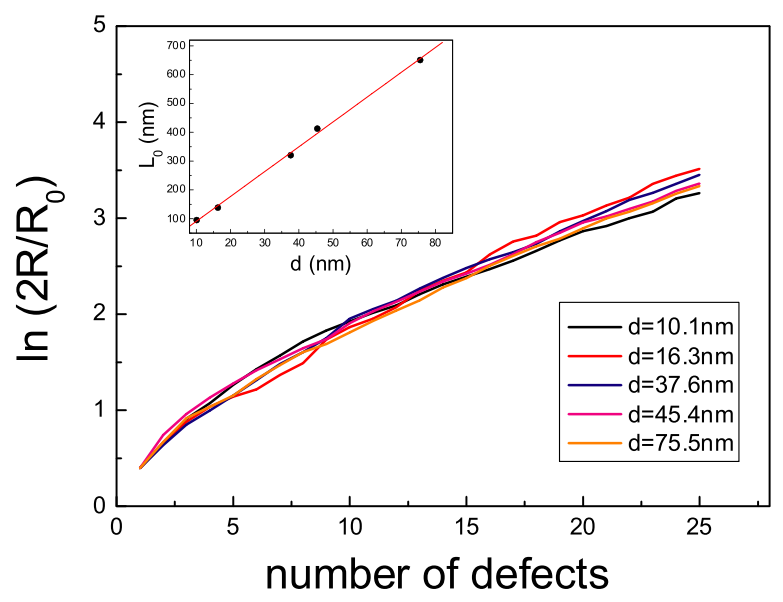

FIG. 3 (color online). Calculated room temperature resistance for different average distances between divacancies $(d)$ as a function of number of defects. Inset: localization length, $L_{0}$, extracted from the fitting $R=R_{0} \times \exp \left(L / L_{0}\right)$ as a function of $d$. localization regime for a very low density of defects, i.e., with more than 5-9 divacancies, provided the resulting $L_{0}$ is smaller than $L_{\phi}$. We have looked into the origin of the reduction in the number of channels in our system by diagonalizing the transmission matrix [18] used in our calculations. The diagonalization supports the previous discussion, although the full reduction to one channel appears only once 5-9 defects have been introduced in the nanotube. Notice that Figs. 1 and 2 already accounted for this result: in Fig. 1, for both 25 or 15 defects the differential conductance is never larger than $G_{0}$; while in Fig. 2, the resistance in this case is never smaller than $R_{0}$ for a number of defects larger than 5 .

The inset of Fig. 3 shows the effective localization length, $L_{0}$, as a function of $d$ for divacancies at room $T$ indicating that $L_{0} \approx 8.5 \mathrm{~d}$. In Ref. [8] we found $L_{0} \approx 4.1 d$ after averaging the resistance in the log scale over many different random cases; note that this is appropriate because of the normal distribution of $\log (R)$ in 1D systems [10]. Our new localization length reflects, however, that at room $T$, and for a particular distribution of divacancies, the conductance of the system is calculated as the sum of the channel contributions associated with the energy window $E_{F} \pm k_{B} T$ (which represents a kind of effective average on energy of the conductance). This new estimation of $L_{0}$ at room $T$ slightly modifies the average distance between divacancies that were estimated in Ref. [8] to have been created in the SWNTs by $\mathrm{Ar}^{+}$, suggesting that those values have to be reduced in all the cases by about $50 \%$. Notice that this density of divacancies was obtained neglecting the effects that monovacancies are producing in the nanotube conductance. This is supported by our calculations for monovacancies whose results can be summarized in the following way: (i) the ballistic regime is found to extend up to about 200 monovacancies; (ii) the localization regime appearing for more than 200 defects shows a resistance as a function of the tube length that can also be fitted to $R_{0} \times$ $\exp \left(L / L_{0}\right)$ with $L_{0} \approx 600 d$, implying that in the localization regime the conductance is again controlled by only one channel. Therefore, monovacancies show a much smaller effect on the nanotube conductance than divacancies do. It is expected that for large vacancy clusters (larger than divacancies), $L_{0}$ would also be proportional to $d$, but with a proportionality constant much smaller than 8.5.

Finally, for the divacancy case we have analyzed the effect of temperature in the quenching of the conductance fluctuations. This has been done for a tube with 25 divacancies as follows. As we are interested in the fluctuations that have already disappeared at room $T$, we take the calculated $R(L)$ at room $T$ as reference data. The scaled resistance plot is shown in the inset of Fig. 4 for $d=$ $75.5 \mathrm{~nm}$ for a particular random configuration [the same as analyzed in Fig. 2(a)], after substraction of its mean value in $L$. We perform this calculation for all random configurations of defects and calculate for each of them the 


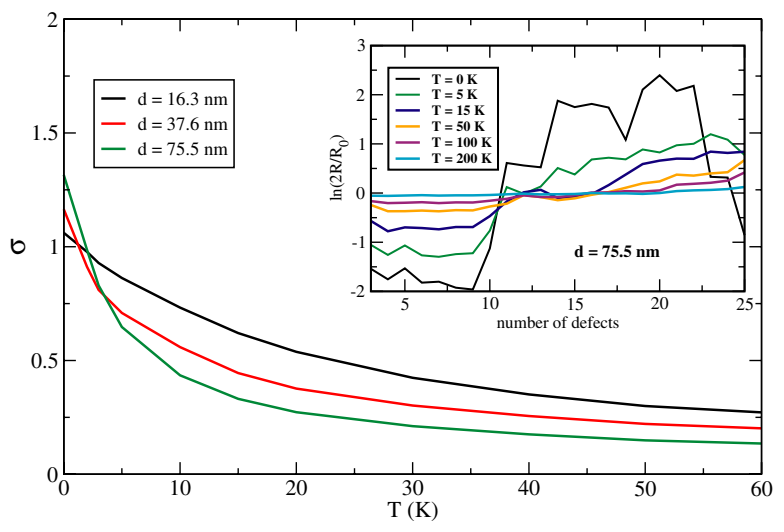

FIG. 4 (color online). Calculated rms deviation $(\sigma)$ as a function of temperature for three different densities of divacancies. The inset shows the calculated resistance referred to the room temperature results for a particular random configuration with $d=75.5 \mathrm{~nm}$ (see text for discussion).

rms deviation $(\sigma)$ with respect to the $R(L)$ data at room $T$. The final average over all configurations of $\sigma$ is plotted in Fig. 4. The general trend is as follows: the higher $T$ is the faster the fluctuation damping. Also, the fluctuations decrease more rapidly for a low density of defects (large $d$ ). Figure 2(a) shows that for $d=75.5 \mathrm{~nm}$, the fluctuations are small for $T$ higher than $T_{C} \approx 15 \mathrm{~K}$, whereas for the other two $d$ 's (not shown here) this occurs for $T_{C} \approx 32$ and $65 \mathrm{~K}$, for $d=37.6$ and $16.3 \mathrm{~nm}$, respectively. Thus, the temperature at which the fluctuations are quenched scales as $1 / d$. This can be understood by noting that at $T_{C}$ the thermal length $L_{T}$ should be of the order of the localization length, $L_{0}$ (that is proportional to $d$ ). Physically, the smaller the density of defects the smaller the averaged scattering potential, therefore a lower $T$ would be needed to wash out the fluctuations. We have also carried out similar numerical calculations for $(5,5)$ metallic SWNTs (not shown here). The underlying physics in this case seems to be the same as the one discussed for $(10,10)$ SWNTs, allowing us to safely conclude that our theoretical findings would remain valid for metallic SWNTs of different chirality.

In conclusion, we have shown that in a $(10,10)$ carbon nanotube: (i) the transition between the ballistic and the localization regimes appears for a small number of divacancies in the nanotube (about 3-5). (ii) For a higher number of defects the system shows localization, reducing the number of effective channels from two (ballistic) to one. (iii) At zero $T$, the conductance of the nanotube as a function of its length shows strong fluctuations. The net effect of a finite $T$ is to wash out the strong fluctuations presented at $T=0 \mathrm{~K}$; our calculations show that those fluctuations could be observed for divacancies (with $d$ smaller than about $70 \mathrm{~nm}$ and $N>5-9$ ) if the temperature is below 15-65 K. We stress here that, in spite of the disappearance of the fluctuations, the exponential behavior of $R(L)$ is still preserved at room $T$. This puts in evidence our assumption of a very low inelastic scattering in SWNTs, i.e., the phase coherence length of electrons much larger than the localization length.

Our results are important in order to understand, in a further study, the high bias conductance where optical phonons do play a key role in limiting the conductance. Still there is a lack of knowledge about the role played by both temperature and defect density in this regime.

The authors were partially supported by the Spanish MCyT under Contract No. MAT2002-01534 and the EC 6th Framework Network of Excellence NANOQUANTA (NMP4-CT-2004-500198). B. B. is indebted to MEC (Spain) for financial support. Computing time for some of these calculations in the Centro de Computación Científica de la UAM is gratefully acknowledged. A. R. acknowledges the Humboldt Foundation under the Bessel research grant (2005). We thank J. Gómez-Herrero for a very intensive and fruitful collaboration.

*Corresponding author.

Electronic address: fj.garcia@uam.es

[1] R. Saito, G. Dresselhaus, and M.S. Dresselhaus, Physical Properties of Carbon Nanotubes (Imperial College Press, London, 1998).

[2] M. Terrones et al., Phys. Rev. Lett. 89, 075505 (2002).

[3] A. Kis et al., Nat. Mater. 3, 153 (2004), and references therein.

[4] A. Javey et al., Nature (London) 424, 654 (2003); J. Park et al., Nano Lett. 4, 517 (2004); A. Bachtold et al., Nature (London) 397, 673 (1999).

[5] S. Latil et al., Phys. Rev. Lett. 92, 256805 (2004); H. Choi et al., Phys. Rev. Lett. 84, 2917 (2000); D. Orlikowski et al., Phys. Rev. B 61, 14194 (2000); V. Crespi, M. L. Cohen, and A. Rubio, Phys. Rev. Lett. 79, 2093 (1997).

[6] F. Banhart, Rep. Prog. Phys. 62, 1181 (1999).

[7] J. Kong et al., Phys. Rev. Lett. 87, 106801 (2001).

[8] C. Gómez-Navarro et al., Nat. Mater. 4, 534 (2005).

[9] A. V. Krasheninnikov et al., Phys. Rev. B 65, 165423 (2002); 63, 245405 (2001).

[10] J. B. Pendry, Adv. Phys. 43, 461 (1994).

[11] S. Datta, Electronic Transport in Mesoscopic Systems (Cambridge University Press, Cambridge, 1997).

[12] F. J. García-Vidal, F. Flores, and S. G. Davison, Prog. Surf. Sci. 74, 177 (2003).

[13] A. A. Demkov et al., Phys. Rev. B 52, 1618 (1995).

[14] At low bias, acoustic phonons are the only source of scattering for an otherwise perfect nanotube. The meanfree path is very long, which makes this dephasing mechanism not relevant for our study in which the interdefect distance is much smaller.

[15] This analysis does not support the model of a tube consisting of series of quantum dots. If this were the case, $L_{0}$ would be just smaller than $d$; as shown in this Letter, this is not the case.

[16] S. Frank et al., Science 280, 1744 (1998).

[17] S. Sanvito et al., Phys. Rev. Lett. 84, 1974 (2000).

[18] P. Jelinek et al., Phys. Rev. B 68, 085403 (2003). 